\title{
The Outcome of Post-Chemotherapy Retroperitoneal Lymph Node Dissection in Patients with Metastatic Bladder Cancer in the Retroperitoneum
}

\author{
Nick W. Liu ${ }^{\mathrm{a}}$, Katie S. Murray ${ }^{\mathrm{b}}$, S. Machele Donat ${ }^{\mathrm{c}}$, Harry W. Herr ${ }^{\mathrm{c}}$, Bernard H. Bochner ${ }^{\mathrm{c}}$ \\ and Guido Dalbagni ${ }^{\mathrm{c}, *}$ \\ a Department of Urology, SUNY Upstate Medical University, Syracuse, NY, USA \\ ${ }^{\mathrm{b}}$ Department of Surgery, Urology Division, University of Missouri School of Medicine, Columbia, MO, USA \\ ${ }^{\mathrm{c}}$ Department of Surgery, Urology Service, Memorial Sloan Kettering Cancer Center, New York, NY, USA
}

Received 23 May 2018

Accepted 13 October 2018

\begin{abstract}
.
Purpose: While a definitive cure can be achieved by radical cystectomy and pelvic lymph node dissection in select patients with regional lymphadenopathy, the benefit remains uncertain in patients who present with non-regional metastases. We analyzed the survival outcomes of post-chemotherapy retroperitoneal lymph node dissection.

Materials and Methods: We reviewed our institutional database and identified 13 patients with radiographically evident or biopsy proven retroperitoneal nodal metastases with a significant response to chemotherapy. These patients underwent consolidative surgery with concomitant or delayed retroperitoneal lymph node dissection. The primary endpoints were progression-free survival and disease-specific survival from the time of retroperitoneal lymph node dissection.

Results: All patients had primary urothelial cell carcinoma. Twelve patients underwent concomitant radical cystectomy, pelvic and retroperitoneal lymph node dissection. Seven patients $(54 \%)$ had residual disease in the retroperitoneum and the median number of retroperitoneal nodes containing metastases was 4 (IQR 2-6). Six (86\%) developed disease recurrences within 2 years of surgery and $5(71 \%)$ died of cancer. Of the 6 patients without residual disease in the retroperitoneum, 2 (33\%) developed recurrences and died of disease progression. The 2-year disease-specific survival was worse for patients with residual disease in the retroperitoneum than those without residual retroperitoneal disease (34\%, 95\% CI 5-68 vs 50\%, 95\% CI 6-85).

Conclusions: The presence of retroperitoneal nodal metastases at post-chemotherapy retroperitoneal lymph node dissection is a poor prognosticator. Consolidative surgery with retroperitoneal lymph node dissection provides important prognostic information and may be therapeutic in a very small subset of these patients.
\end{abstract}

Keywords: Surgery

ABBREVIATIONS AND ACRONYMS
$\begin{array}{ll}\text { UC } & \text { urothelial carcinoma } \\ \text { PC-RPLND } & \begin{array}{l}\text { post-chemotherapy retroperitoneal } \\ \text { lymph node dissection }\end{array} \\ \text { PFS } & \begin{array}{l}\text { progression-free survival } \\ \text { cancer-specific survival }\end{array} \\ \text { CSS } & \end{array}$

\footnotetext{
${ }^{*}$ Correspondence to: Guido Dalbagni, MD, Department of Surgery Urology Service, Memorial Sloan Kettering Cancer Center, 1275 York Ave, New York, NY, 10065 USA. Tel.: +1 646422 4394; Fax: +1 212988 0760; E-mail: dalbagng@ mskcc.org.
} 


\section{INTRODUCTION}

Cisplatin-based chemotherapy is the mainstay treatment for metastatic UC of the bladder and modern chemotherapy regimens have resulted in overall response rates of $50 \%$ to $70 \%$, including a complete response rate of $20 \%$ to $30 \%$ [1,2]. Despite an impressive overall response rate, long-term survival remains poor with only a small portion of patients surviving beyond five years [3]. Most referral centers have adopted a multimodal approach for the treatment of metastatic bladder cancer and consider surgical consolidation for patients with significant response to chemotherapy. Surgical consolidation is most commonly done in patients who present initially with unresectable pelvic disease or regional metastases. However, surgical resection of distant nodal disease or visceral metastases has been described as well [4-6].

The rationale for post-chemotherapy surgery is that disease relapse most frequently occurs at responding sites of disease even in patients with complete response to chemotherapy [7]. In light of this finding, one may assume that surgical resection of the initial site of disease may improve survival outcome in these patients. However, the data on this approach is limited and, currently, there is no clear indication for postchemotherapy surgery in patients with metastatic UC. Previous reports from our institution have shown that post-chemotherapy surgical resection of locoregional disease is beneficial in patients exhibiting significant response to chemotherapy, but the benefit of surgery in distant nodal disease is less clear $[8,9]$.

The objective of this study is to describe the impact of PC-RPLND in a contemporary cohort of patients who exhibited a significant response to modern chemotherapy regimen for metastatic bladder cancer.

\section{MATERIALS AND METHODS}

Following institutional board approval, we conducted a retrospective review of patients with metastatic UC of the bladder treated with systemic chemotherapy at our institution between 2002 and 2014. Of these, we selected patients with infrarenal retroperitoneal nodal disease above the aortic bifurcation who developed a significant response to chemotherapy. Eligibility criteria included patients who presented with either retroperitoneal nodal metastases at diagnosis or solitary relapse in the retroperitoneum after initial radical cystectomy. Patients with visceral metastases at the time of diagnosis or at relapse were excluded from the study. Preoperative lymph node biopsy to confirm presence of metastases was not a requirement for this study. Thirteen patients met these criteria and were included in this study.

The standard chemotherapy regimen for metastatic $\mathrm{UC}$ at our institution is 6 cycles of gemcitabine and cisplatin. However, alternative regimens were used based on patient comorbid conditions, prior history of chemotherapy administration, and chemotherapy toxicity and tolerability throughout treatment. Following chemotherapy, response was assessed via MRI, CT or PET-imaging. Complete response was defined as resolution of all measureable disease in the retroperitoneum for a minimum of 4 weeks after completion of chemotherapy. Partial response was defined as a $50 \%$ decrease in all measureable disease.

The boundary of PC-RPLND was at the discretion of the surgeon at the time of surgery but always included the site of disease in the retroperitoneum seen on pre-chemotherapy imaging. The extent of dissection included full bilateral (to the level of renal vein bilaterally), paraaortic (to the level of left renal vein), interaortocaval/para-caval (to the level of right renal vein) and low-retroperitoneal (bilateral dissection to the level of the inferior mesenteric artery). Pathologic analysis included pathologic stage, presence of variant histology, total number of nodes removed and the number and location of nodal metastases.

After surgery, patients were followed up with serial chest and abdominal imaging at 3 to 6-month intervals. Patients with progression of disease were eligible for additional chemotherapy or clinical trials at our institution. Progression free-survival was defined as time of RPLND to first discovery of local recurrence or distant metastasis. Cancer specificsurvival was calculated as time from RPLND to death from disease. Survival analysis was done using the Kaplan-Meier method. Statistical analyses were performed using Stata ${ }^{\circledR}$ version 13 (StataCorp, College Station, Texas) with $p$ values $<0.05$ considered statistically significant.

\section{RESULTS}

\section{Patient characteristics}

The median age at PC-RPLND was 67 years (IQR 59-70). Seven patients (54\%) were male. 
Eight patients (62\%) had preoperative biopsy of either retroperitoneal or pelvic lymph nodes for confirmation of metastatic disease. Table 1 lists the clinical characteristics and oncological outcomes of the cohort. All patients in the study completed 6 cycles of systemic chemotherapy at the time of diagnosis. Nine patients $(69 \%)$ completed the standard regimen of gemcitabine and cisplatin. Two patients who underwent delayed PC-RPLND received neoadjuvant gemcitabine and cisplatin prior to radical cystectomy. Two patients were treated with an alternative chemotherapy regimen because of intolerability to cisplatin. Radiographic complete response was achieved in 8 patients $(62 \%)$ prior to postchemotherapy surgery.

\section{Perioperative and pathological characteristics}

Eleven patients ( $85 \%$ ) underwent concomitant radical cystectomy, pelvic dissection and retroperitoneal lymph node dissection. Two patients (15\%) underwent neobladder reconstruction while the others had ileal conduit urinary diversion. For these 11 patients, the median estimated blood loss was $900 \mathrm{~mL}$ (IQR 500-1200) and the median operative time was 373 minutes (IQR 301-457). There were no perioperative deaths within 30 days.

Five patients had full bilateral RPLND along with pelvic lymph node dissection. The median number of retroperitoneal and pelvic lymph nodes removed were 8 (IQR 7-11) and 18 (IQR 12-25), respectively. Seven patients $(50 \%)$ had viable disease in the retroperitoneal nodes and the median number of retroperitoneal nodes containing metastases was 4 (IQR 2-6). When stratifying patients based on presence of residual disease in the retroperitoneum, the incidence of complete clinical response was higher in patients without residual disease than those with residual disease ( $83 \%$ vs $43 \%$ ).

\section{Oncologic outcome}

At a median follow up of 24 months (IQR 6-56), 8 patients $(62 \%)$ developed disease recurrences and 7 (50\%) died of disease progression. Three patients recurred in the retroperitoneum outside the field of initial dissection, 6 patients developed distant metastases (outside of retroperitoneum) and 1 patient with both local and distant disease. The median PFS and CSS rates for the entire cohort were 14 and 21 months, respectively. The 2-year PFS and CSS rates were 31\% and $42 \%$, respectively (see Fig. 1). Of the 7 patients with residual disease in the retroperitoneum, $6(85 \%)$ developed disease recurrences within two years of PC-RPLND and 5 (71\%) died of disease progression. Four patents developed visceral or distant metastases while two recurred within the retroperitoneum. Two patients (29\%) are alive at the most recent follow up but only 1 patient is alive without evidence of disease at 24 months after PC-RPLND in this group. In patients without residual retroperitoneal disease at PC-RPLND, 2 (33\%) patients developed disease recurrence and subsequently died of disease progression. Both patients had residual disease in the pelvic nodes at the time of surgery and both recurred outside the retroperitoneum after surgery. The 2-year diseasefree survival was worse for patients with residual disease in the retroperitoneum than those without residual retroperitoneal disease $(34 \%, 95 \%$ CI 5-68 vs $50 \%, 95 \%$ CI 6-85).

\section{DISCUSSION}

While the benefit of surgical resection of lymph nodes in patients with regional pelvic lymphadenopathy is well established, the benefit of post-chemotherapy surgery in patients with distant nodal metastases remains unclear [10]. We report a 2-year disease-free survival of $42 \%$ in patients with metastatic bladder cancer of the retroperitoneal lymph nodes treated with PC-RPLND. When patient outcomes were stratified by presence of viable nodal disease, our study demonstrated that the presence of residual disease in the retroperitoneum at PCRPLND is a poor prognostic indicator. The survival outcome is worse in patients with residual disease in the retroperitoneum than those without residual disease. Despite this sobering finding, we believe PCRPLND in this setting may be therapeutic for a small subset of patients who otherwise would succumb to recurrent urothelial carcinoma. In the current series, 1 out of $7(14 \%)$ patients with residual disease is alive without evidence of disease at 2 years after surgical consolidation.

Previous reports evaluating the outcomes of postchemotherapy surgery in patients with metastatic bladder cancer involving the retroperitoneum is in line with our findings. The study by Dodd et al. revealed that, while patients with regional adenopathy had improved outcomes with post-chemotherapy surgery, those with distant lymph nodal disease had poorer prognosis despite surgical resection [8]. In a subgroup analysis of 203 patients with 


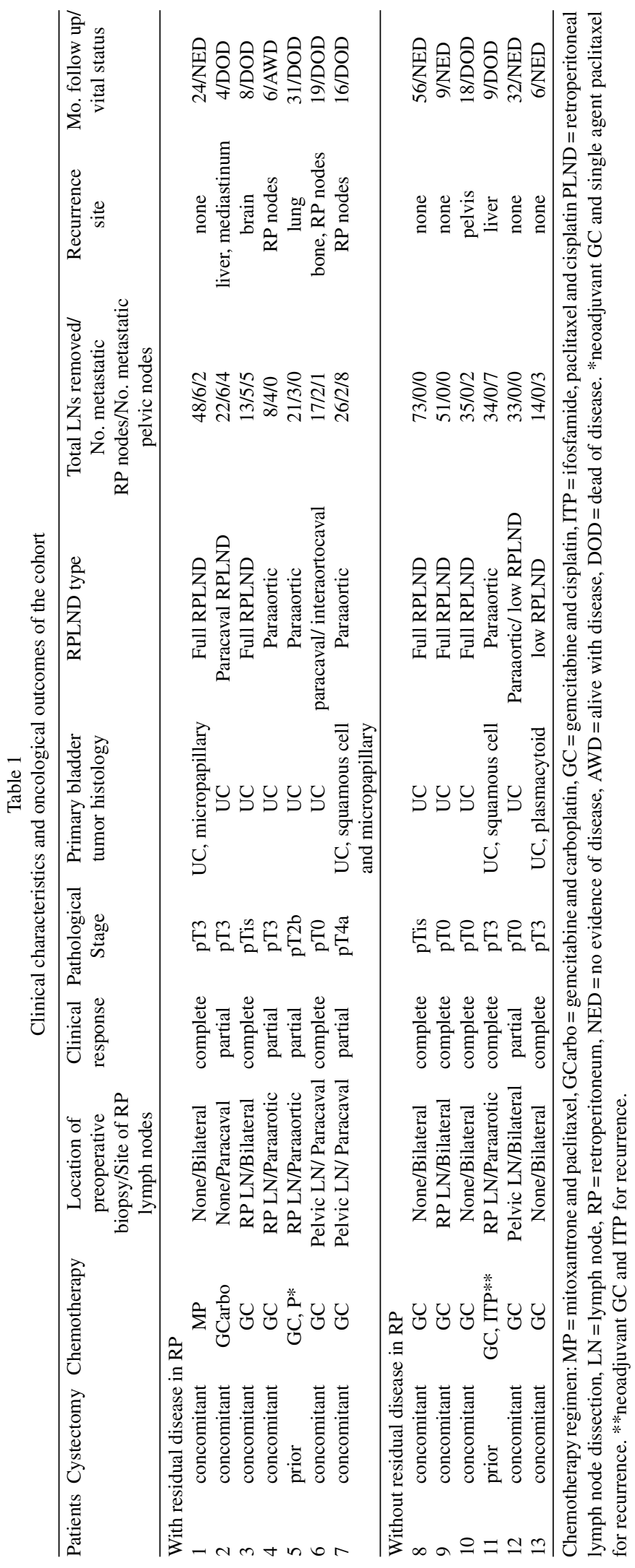



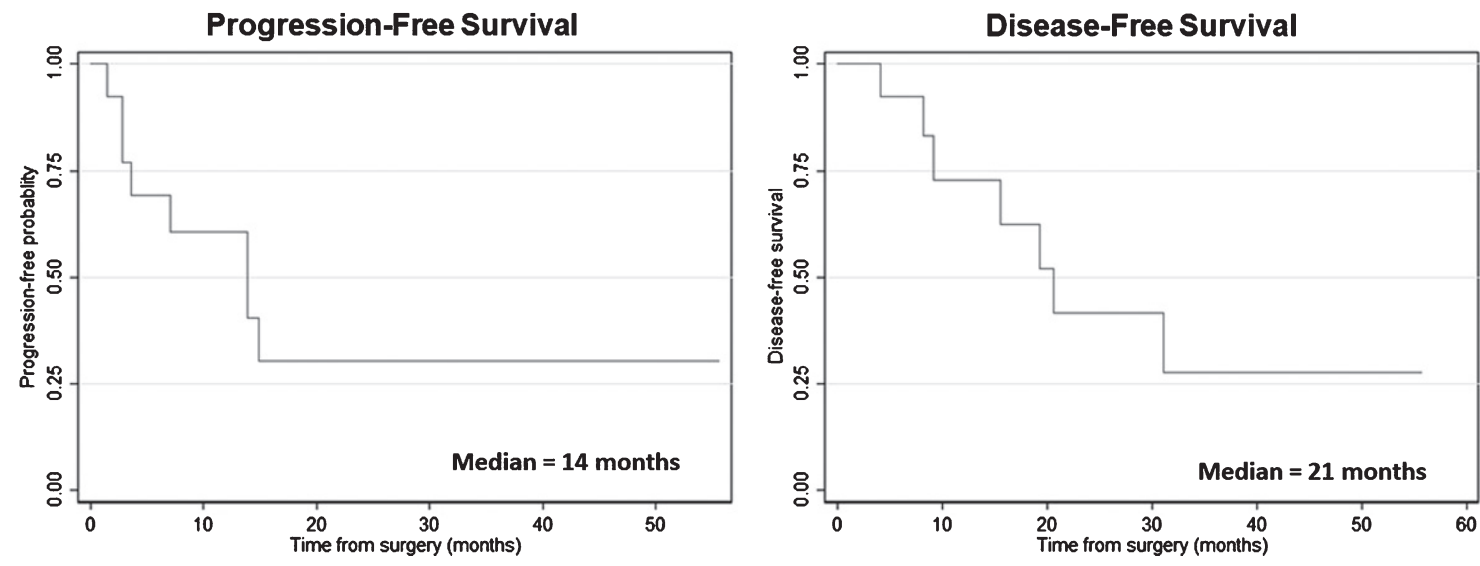

Fig. 1. Kaplan-Meier survival plots for cancer-specific survival for the entire cohort.

unresectable or metastatic bladder cancer who had post-chemotherapy surgeries, the authors reported on a heterogeneous group of 11 patients with nonregional or distant lymph node disease, including 8 patients with retroperitoneal lymph nodal involvement. Only 2 patients in this group (18\%) were alive at 5 years. Similarly, in a series of 11 patients with biopsy proven metastatic bladder cancer involving retroperitoneal lymph nodes treated with full bilateral PC-RPLND, the investigators from MD Anderson Cancer Center demonstrated that none of the patients with viable tumor involving more than two retroperitoneal lymph nodes were alive at 2 years after surgery [11].

These findings bring into question whether consolidative surgeries in patients with metastatic retroperitoneal nodal disease is truly advantageous. While patients with residual retroperitoneal nodal disease portend a poor prognosis, those with 2 or less retroperitoneal lymph nodes treated at MD Anderson enjoyed a significantly improved survival, suggesting that eliminating residual disease may cure a small group of these patients especially in those with low residual disease burden [11]. In a retrospective review of 14 patients with supra-regional metastases treated with neoadjuvant cisplatin-based chemotherapy followed by consolidative surgeries including complete RPLND, the investigators from the Netherlands demonstrated a durable long-term survival of $36 \%$ and $24 \%$ at 3 and 5 years, respectively [12]. At least 1 out of 5 patients (20\%) with residual nodal disease in this study lived beyond 3 years after consolidative surgery. Similarly, our study found that long-term response can be achieved with consolidative surgical resection in a small group of patients with residual nodal disease. In the absence of a randomized trial comparing chemotherapy with and without surgery, it remains difficult to determine the true benefit of consolidative surgery in this setting. However, based on these findings, it is reasonable to offer combined modality treatment in patients presenting with retroperitoneal nodal metastases amenable to safe and complete surgical resection. Furthermore, RPLND in post-chemotherapy setting can provide important prognostic information and may help direct further therapeutic intervention in this cohort.

What selection criteria can we use to identify patients who would benefit from consolidative surgery with PC-RPLND? Unfortunately, the current study is too small to define any meaningful prognostic factors to identify patient subgroups likely to benefit from PC-RPLND. With that said, most centers of excellence would agree that having a significant chemotherapy response is a prerequisite for post-chemotherapy surgery. Previous reports evaluating patients with metastatic UC of the bladder found that chemotherapy response status was an important prognostic indicator of outcome. The study by Herr et al. noted that while a third of patients undergoing post-chemotherapy surgery were alive at 5 years, post-chemotherapy surgery did not benefit those who failed to achieve a significant response to chemotherapy [9].

Additionally, in a recent report evaluating the long-term efficacy of post-chemotherapy lymphadenectomy in patients with metastatic urothelial cancer of the upper tract and bladder, Necchi et al. found that complete clinical response to chemotherapy was predictive of recurrence-free survival [13].

In addition to chemotherapy response, previous studies have shown that metastatic disease burden, sites of metastases and patient performance status are 
all important prognostic determinants for outcomes of surgical consolidation [3, 4, 14]. Based on these findings, patients with bulky retroperitoneal lymph nodes after chemotherapy should be treated with second-line chemotherapy or investigational clinical trial rather than aggressive surgical resection. In the setting of metastatic UC involving the retroperitoneal lymph nodes, our institution would consider consolidative RPLND if the following criteria are met: 1) a complete response to systemic chemotherapy, 2) limited nodal metastases amenable to complete surgical resection, and 3) patients with good performance status [15].

Several important limitations of this study are noteworthy. First, it was retrospective in nature and included a relatively small number of patients from a single institution, which may limit its external validity. Second, preoperative biopsy of retroperitoneal lymph nodes confirming metastases was not performed in all patients, thus our study may have included patients without true metastatic disease. Third, a standardized RPLND template was not used in this study and the extent of lymph node dissection was based on surgeon discretion, thus making the result of the study difficult to interpret. However, to our knowledge, there is no data supporting the use of a standardized RPLND template for the treatment of metastatic bladder cancer. Finally, the survival benefit of lymphadenectomy seen in the current study may be a reflection of selection bias rather than a true therapeutic benefit derived from surgery itself. In other solid organ tumors, adjunct regional lymphadenectomy has not been consistently shown to improve survival $[16,17]$. Even in bladder cancer, no level 1 evidence exists to address the value of the extent of regional lymphadenectomy. The preliminary finding of a recent randomized trial from Germany (LEA22) comparing the outcomes of extended vs standard pelvic LND only showed a survival trend favoring extended lymphadenectomy but the difference was not statistically significant [18]. To truly define the best treatment strategy for patients with metastatic bladder cancer of the retroperitoneum in the postchemotherapy setting, a prospective trial involving multiple experienced centers and a standardized surgical template is needed.

\section{CONCLUSION}

The presence of retroperitoneal nodal metastases at PC-RPLND is a poor prognosticator. Consolidative surgery with RPLND provides important prognostic information and may be therapeutic in an extremely small subset of these patients. A prospective study with larger sample size is needed to fully address this question.

\section{CONFLICTS OF INTEREST}

None of the authors have any conflict of interests.

\section{FUNDING SOURCES}

The Sidney Kimmel Center for Prostate and Urologic Cancers and the National Institutes of Health/National Cancer Institute Cancer Center Support Grant, award number P30 CA008748.

\section{REFERENCES}

[1] Sternberg CN, de Mulder PH, Schornagel JH, et al. Randomized phase III trial of high-dose-intensity methotrexate, vinblastine, doxorubicin, and cisplatin (MVAC) chemotherapy and recombinant human granulocyte colony-stimulating factor versus classic MVAC in advanced urothelial tract tumors: European Organization for Research and Treatment of Cancer Protocol no. 30924. J Clin Oncol. 2001;19:2638.

[2] von der Maase H, Sengelov L, Roberts JT, et al. Longterm survival results of a randomized trial comparing gemcitabine plus cisplatin, with methotrexate, vinblastine, doxorubicin, plus cisplatin in patients with bladder cancer. J Clin Oncol. 2005;23:4602.

[3] Bajorin DF, Dodd PM, Mazumdar M, et al. Long-term survival in metastatic transitional-cell carcinoma and prognostic factors predicting outcome of therapy. J Clin Oncol. 1999; 17:3173.

[4] Abe T, Shinohara N, Harabayashi T, et al. Impact of multimodal treatment on survival in patients with metastatic urothelial cancer. Eur Urol. 2007;52:1106.

[5] Lehmann J, Suttmann H, Albers P, et al. Surgery for metastatic urothelial carcinoma with curative intent: The German experience (AUO AB 30/05). Eur Urol. 2009;55:1293.

[6] Otto T, Krege S, Suhr J, et al. Impact of surgical resection of bladder cancer metastases refractory to systemic therapy on performance score: A phase II trial. Urology. 2001;57:55.

[7] Dimopoulos MA, Finn L, Logothetis CJ. Pattern of failure and survival of patients with metastatic urothelial tumors relapsing after cis-platinum-based chemotherapy. J Urol. 1994;151:598.

[8] Dodd PM, McCaffrey JA, Herr H, et al. Outcome of postchemotherapy surgery after treatment with methotrexate, vinblastine, doxorubicin, and cisplatin in patients with unresectable or metastatic transitional cell carcinoma. J Clin Oncol. 1999;17:2546.

[9] Herr HW, Donat SM, Bajorin DF. Post-chemotherapy surgery in patients with unresectable or regionally metastatic bladder cancer. J Urol. 2001;165:811.

[10] Yafi FA, Kassouf W. Management of patients with advanced bladder cancer following major response to systemic chemotherapy. Expert Rev Anticancer Ther. 2009;9:1757. 
[11] Sweeney P, Millikan R, Donat M, et al. Is there a therapeutic role for post-chemotherapy retroperitoneal lymph node dissection in metastatic transitional cell carcinoma of the bladder? J Urol. 2003;169:2113.

[12] de Vries RR, Nieuwenhuijzen JA, Meinhardt W, et al. Long-term survival after combined modality treatment in metastatic bladder cancer patients presenting with supraregional tumor positive lymph nodes only. Eur J Surg Oncol. 2009;35:352.

[13] Necchi A, Giannatempo P, Lo Vullo S, et al. Postchemotherapy lymphadenectomy in patients with metastatic urothelial carcinoma: Long-term efficacy and implications for trial design. Clin Genitourin Cancer. 2015;13:80.

[14] Abe T, Kitamura H, Obara W, et al. Outcome of metastasectomy for urothelial carcinoma: A multi-institutional retrospective study in Japan. J Urol. 2014;191:932.
[15] Herr HW. Is metastasectomy for urothelial carcinoma worthwhile? Eur Urol. 2009;55:1300.

[16] Coit D. The Enigma of Regional Lymph Nodes in Melanoma. N Engl J Med. 2017;376:2280.

[17] Gervasoni JE Jr, Sbayi S, Cady B. Role of lymphadenectomy in surgical treatment of solid tumors: An update on the clinical data. Ann Surg Oncol. 2007;14:2443.

[18] Gschwend JE, Heck MM, Lehmann J, et al. Limited versus extended pelvic lymphadenectomy in patients with bladder cancer undergoing radical cystectomy: Survival results from a prospective, randomized trial (LEA AUO AB 25/02). J Clin Oncol. 2016;34:4503. 